\title{
O.R. Applications
}

\section{The role of retailer's performance in optimal wholesale price discount policies}

\author{
Igor Bykadorov $^{\mathrm{a}}$, Andrea Ellero $^{\mathrm{b}, *}$, Elena Moretti $^{\mathrm{b}}$, Silvia Vianello $^{\mathrm{c}}$ \\ ${ }^{a}$ Sobolev Institute of Mathematics, Siberian Branch of the Russian Academy of Sciences, 4 Acad. Koptyug Avenue, 630090 Novosibirsk, Russia \\ ${ }^{\mathrm{b}}$ Department of Applied Mathematics, University Ca' Foscari of Venice, Dorsoduro 3825/E, 30123 Venezia, Italy \\ ${ }^{\mathrm{c}}$ Department of Business and Management Studies, University Ca' Foscari of Venice, Cannaregio 873, 30121 Venezia, Italy
}

Received 23 January 2007; accepted 6 December 2007

Available online 28 January 2008

\begin{abstract}
The main goal of this paper is to model the effects of wholesale price control on manufacturer's profit, taking explicitly into account the retailer's sales motivation and performance. We consider a stylized distribution channel where a manufacturer sells a single kind of good to a single retailer. Wholesale price discounts are assumed to increase the retailer's motivation thus improving sales. We study the manufacturer's profit maximization problem as an optimal control model where the manufacturer's control is the discount on wholesale price and retailer's motivation is one of the state variables. In particular in the paper we prove that an increasing discount policy is optimal for the manufacturer when the retailer is not efficient while efficient retailers may require to decrease the trade discounts at the end of the selling period. Computational experiments point out how the discount on wholesale price passed by the retailer to the market (passthrough) influences the optimal profit of the manufacturer.
\end{abstract}

(C) 2007 Elsevier B.V. All rights reserved.

Keywords: Wholesale price; Sales motivation; Optimal control

\section{Introduction}

Manufacturers have various ways to encourage increased sales acting on retailers with wholesale price reductions, slotting fees, buydowns, and so on. The effects of these marketing tools on sales are filtered by the retailer's sales attitude and aptitude. Retailer motivation level, sales ability and his role perception are the main mediating variables between manufacturers incentives and retailer's sales performance (Walker et al., 1977; Churchill et al., 1985): roughly speaking the expected outcomes of manufacturer's actions depend both on the nature of the retailer (his skill, role perception, aptitude) and on retailer's sale motivation, his engagement and zeal.

The main goal of this paper is to model the effects of the main push strategy, wholesale price control (Walters, 1989), on retailer's motivation and on manufacturer's profit, bearing in mind that such effects will depend on retailer's personal features and skill. Wholesale price promotions that may favorably drive retailer's motivation, are considered as a dynamic process spread over a finite selling period. We propose an optimal control model in which trade discounts flow along a stylized vertical distribution channel where a single manufacturer serves a single market, through a single retailer, with the aim to maximize her profit.

The optimal control model we propose recalls similar dynamic models proposed in the quantitative marketing literature. The role of advertising and, more in general, of communication in marketing has been largely explored by means of

\footnotetext{
${ }^{*}$ Corresponding author. Tel.: +39 0412346930; fax: +39 0415221756 .

E-mail addresses: bykad@math.nsc.ru (I. Bykadorov), ellero@unive.it (A. Ellero), emoretti@unive.it (E. Moretti), silviav@unive.it (S. Vianello).
} 
dynamic and optimal control models, often considering advertising goodwill as a mean to store the effects of advertising efforts (see e.g. Seierstad and Sydsæter, 1987; Feichtinger et al., 1994; Sethi and Thompson, 2000; Jørgensen and Zaccour, 2004). In a seminal paper, Nerlove and Arrow (see Nerlove and Arrow, 1962) take into account the role of the goodwill of a product explicitly: in a rather similar way we consider explicitly motivation as a mean to accumulate the effects of wholesale promotion activity of the manufacturer.

A general definition of motivation was given in Mitchell (1982) as "the degree to which an individual wants and chooses to engage in certain specified behaviors", it turns out that motivation cannot be assimilated with behavior and outcome performance (see also Miao et al., 2007; Grant et al., 2001). Two main types of motivation are usually distinguished: extrinsic and intrinsic motivation. Retailer's motivation is extrinsic if he can satisfy his needs indirectly, for instance with monetary compensation (Osterloh and Frey, 2000), like trade discounts.

On the other hand, motivation is intrinsic if the retailer can satisfy his needs directly, that is when he acts for an immediate need of satisfaction (Osterloh and Frey, 2000). Intrinsically motivated individuals do something for the satisfaction derived from the activity itself, they perform behaviors not for money but for their own sake.

The retailer's behavior is influenced by his sales motivation, intrinsic and extrinsic (Walker et al., 1977; Churchill et al., 1985; Miao et al., 2007): for instance, if the retailer is motivated he can decide to place the manufacturer's products in a strategic location around the store, he can help to achieve the manufacturer's market goals, he can provide after sale support and so on.

The goal of the manufacturer is above all to maximize her profit, for this reason she has to encourage any retailer's behavior that helps to achieve this goal, trying to enhance his job performance, and performances are "partially determined by motivation to work hard and, therefore, increases in motivation should result in greater effort and higher performance" (Mitchell, 1982). Trade discounts can be offered to retailers expecting that they will lead to an increased sales motivation level, obtaining this way an improvement of their sales performance as an indirect effect of trade promotions on sales. But manufacturers expect also that a part of the discount is passed-through along the channel so as to offer a reduced shelf price to the consumers. In particular we consider a retailer who sells at a final price computed using a fixed pass-through percentage.

The price reduction accorded to the final consumer affects sales, of course: this way we have a direct effect of trade discount on sales. ${ }^{1}$

The manufacturer's profit maximization problem is formulated in Section 2 as an optimal control problem where the manufacturer's control is the trade discount. In Section 3 we provide the solution of the profit maximization problem and some numerical examples. Sensitivity analysis with respect to the retailer's skill and to pass-through is provided in Section 4. A final discussion, in Section 5, provides some comments on the main results obtained, on the shortcomings of the model and some suggestions for future research. The proofs involve a lot of technicalities and are reported in the Appendices.

\section{Formulation of the model}

We consider a stylized distribution channel with a manufacturer who sells a single kind of good during a limited time period $\left[t_{1}, t_{2}\right]$, e.g. it is a seasonal product, and assume that the manufacturer's aim is to maximize her total profit in the given time period. The manufacturer supplies a single downstream firm, the retailer.

Transportation, ordering and repricing are considered as done instantaneously, moreover stocks do not exist and information is perfect and with no delays. This assumption may be seen as overly simplistic since the effects of delays and imperfect information have been proved to be appreciable, leading to rigidity and asymmetry in pricing (Ray et al., 2006), bullwhip effects (Ouyang and Daganzo, 2006) and so on. Nevertheless these simplifications are useful to obtain some qualitative insights into the incentive process which may be not easy to be obtained with more sophisticated models.

We assume that the manufacturer can drive the channel behavior offering trade discounts. His goal is to increase this way sales motivation of the retailer, i.e. to stimulate retailer's behavior oriented toward raising the customer's demand. In fact, although sales depend on the retailer's skill, even the best retailer must be motivated to sell the product. For example if he is satisfied with price discounts he will help in selling the product. Moreover the retailer could, and we assume he does, transfer a part of the discount to the consumer (pass-through) selling at a reduced price and this increases sales too. A price discount thus, affects both consumers' demand and retailer's sales motivation.

Trade price discounts reduce the unit profit of the manufacturer so it becomes crucial to pay attention in determining the correct amount of discount and to change it depending on the dynamic behavior of sales. To do this we have to take into account the relationship among retailer's motivation, sales and trade discount. As mentioned before, a trade discount, at

\footnotetext{
${ }^{1}$ Push strategies, like wholesale price reductions are not always optimal, in particular for small manufacturers. When a manufacturer faces a giant retailer wholesale price reductions may be pocketed by retailers who are the leaders of the channel (Gerstner and Hess, 1995) or may lead to cross-brand pass-through (Besanko et al., 2005). But here we assume that the manufacturer is the leader of the channel, the retailer is small.
} 
least if sufficiently high, improves the motivation to sell, while we assume that low trade discounts decrease sales motivation. We also expect that motivation to sell depends on profit which, in turn, depends on cumulative sales.

Let us now go into some more detail and consider an optimal control model in which the cumulative sales and the retailer's motivation are the state variables while the manufacturer controls the wholesale price.

In the following, $x(t)$ denotes the cumulative sales in the time period from $t_{1}$ until $t$. The derivative of the cumulative sales, $\dot{x}(t)$, represents the sales intensity at time $t$. If we denote with $w(t)$ the wholesale price at time $t$ then the profit of the manufacturer during the sale period is given by:

$$
\int_{t_{1}}^{t_{2}}\left(w(t)-c_{0}\right) \dot{x}(t) \mathrm{d} t
$$

where $c_{0}$ is the unit production cost, assumed to be constant. To highlight trade discount we write the wholesale price $w(t)$ as $w(t)=p(1-\alpha(t))$, where $p$ is the wholesale list price and $\alpha(t)$ is the discount on that price granted by the manufacturer at time $t$. Since sales begin at $t_{1}$, i.e. $x\left(t_{1}\right)=0$, the profit reads:

$$
\left(p-c_{0}\right) x\left(t_{2}\right)-p \int_{t_{1}}^{t_{2}} \dot{x}(t) \alpha(t) \mathrm{d} t .
$$

We assume that the trade discount will never be so high as to let the manufacturer's profit become negative: as it will be discussed in detail in Section 3, this leads to a box constraint on the control variable $\alpha(t) \in\left[\alpha_{1}, \alpha_{2}\right] \subseteq[0,1]$.

The second state variable, $M(t)$, sums up the retailer's motivation at time $t$.

We assume that the retailer's motivation is proportional to his outcome (present and past), measured by the cumulative sales, and to the satisfaction of his expectation of a fair reward (see Walters, 1989; Mitchell, 1982).

More precisely we assume that a high level of trade discount, say higher than a fixed level $\bar{\alpha}$, pushes upwards sales motivation of the retailer, but a discount that is underrewarding, say it is lower than $\bar{\alpha}$, decreases motivation. The threshold $\bar{\alpha}$ represents the trade discount expected by the retailer (we assume $\alpha_{1}<\bar{\alpha}<\alpha_{2}$, i.e. the minimum expected trade discount guarantees a positive profit to the manufacturer).

Summarizing the above assumptions, we consider the dynamics of the state variable $M(t)$ to be described by the following equation:

$$
\dot{M}(t)=\gamma \dot{x}(t)+\epsilon(\alpha(t)-\bar{\alpha}),
$$

where $\gamma$ and $\epsilon$ are strictly positive constants related to the character or "psychology" of the retailer. The parameter $\gamma$ represents the sensitivity of the retailer to sales, while $\epsilon$ represents his sensitivity to the amplitude of wholesale price discounts.

Observe that we model motivation as depending explicitly from the total sales, which are proportional to retailer's revenue, and from the total discount perceived during the sales interval, which is proportional to his revenue too. As one can expect, thus, we implicitly consider motivation as related to retailer's profit.

Let us turn to the dynamics of the cumulative sales $x(t)$. Sales are influenced by the retailer's performance. In particular motivation can be considered as a predictor of salesperson's performance (Churchill et al., 1985). Therefore, as established by the firm-level ability-motivation paradigm (Boulding and Staelin, 1993) we consider performance as a function of both retailer's motivation and ability, i.e. retailer's skill, aptitude level, understanding of the task, and so on (see also Campbell and Pritchard, 1976). Following Vroom (1964) and Campbell and Pritchard (1976), we assume that performance is proportional to the product of the motivation $M(t)$ and a parameter $\delta$ which summarizes intrinsic, neither rapidly nor easily modifiable, qualities of the retailer, like skill and aptitude. Selling skill in particular, is considered as a pure and direct surrogate of salesperson's abilities (Rentz et al., 2002).

Of course, sales depend on shelf price. To highlight the effect of trade discounts on final price, we observe that shelf prices can be written as a list price minus shelf price discounts, which we assume to be a fixed quota $\beta$ (pass-through) of the trade discount $\alpha(t)$. This way we will describe changes in sales as depending on the product $\beta \alpha(t)$.

In the model we also consider that, depending on the dimension of the target market, a damping saturation effect arises.

We come this way to describe the dynamics of the cumulative sales as:

$$
\dot{x}(t)=-\theta x(t)+\delta M(t)+\tilde{\eta} \beta \alpha(t),
$$

where $\delta$ represents the retailer's selling skill $(\delta>0), \tilde{\eta}$ is the market sensitivity to shelf price discounts $(\tilde{\eta}>0), \beta$ is the constant pass-through (with $\beta \in(0,1)$ ) and $\theta>0$ is a saturation aversion parameter (see e.g. Vidale and Wolfe, 1957): the sales rate decreases as the sales increase, modeling the market saturation effect, and high values of $\theta$ correspond to small markets with a fast saturation effect, while low values of $\theta$ represent wide markets. 
This way the following optimal control problem can be formulated:

$$
\begin{array}{cl}
P: \text { maximize } & \left(p-c_{0}\right) x\left(t_{2}\right)-p \int_{t_{1}}^{t_{2}} \dot{x}(t) \alpha(t) \mathrm{d} t \\
\text { subject to } & \dot{x}(t)=-\theta x(t)+\delta M(t)+\eta \alpha(t), \\
& \dot{M}(t)=\gamma \dot{x}(t)+\epsilon(\alpha(t)-\bar{\alpha}), \\
& x\left(t_{1}\right)=0, \quad M\left(t_{1}\right)=\bar{M}, \\
& \alpha(t) \in\left[\alpha_{1}, \alpha_{2}\right] \subseteq[0,1],
\end{array}
$$

where $\bar{M}>0$ is the initial motivation of the retailer and $\eta=\tilde{\eta} \beta$ is used to simplify notation.

Substituting $\dot{x}(t)$ in the profit function and in the second motion equation the problem can be rewritten in normal form and turns out to be linear with respect to the state variables and quadratic with respect to control.

As maximum allowable discount we consider the value $\alpha_{2}$ such that the unit profit vanishes $p-c_{0}-p \alpha_{2}=0$, i.e.

$$
\alpha_{2}=\left(p-c_{0}\right) / p
$$

We also assume that if the wholesale price is constant during the sale period, i.e. $\alpha(t)$ is constant $\forall t \in\left[t_{1}, t_{2}\right]$, then the cumulative sales function, $x(t)$, is concave. In other words we assume that the market is mature, and a clear saturation effect can be observed. This requires, as one can expect, that the market is not "too" large or, in other words, that the saturation parameter is "sufficiently" high. More precisely (for detailed computations see Appendix A), throughout the paper we will assume

$$
\theta \geqslant\left(\frac{\epsilon\left(\alpha_{2}-\bar{\alpha}\right)}{\delta \bar{M}+\eta \alpha_{2}}+\gamma\right) \delta
$$

which is the necessary and sufficient condition that ensures concavity of the cumulative sales function $x(t)$ in case of constant wholesale price.

\section{Optimal trade discount policy}

The optimal wholesale price reduction that should be allowed to the retailer can be obtained solving problem $P$ by means of optimal control techniques, we do this in detail in Appendix B.

The structure of the optimal trade discount strategy over time turns out to have two essentially different forms depending on the retailer's efficiency, his selling skill, which is described by the model parameter $\delta$. More precisely if $\delta$ is less than a certain threshold, the retailer is rather inexpert or unskilled, and the optimal policy is to progressively increase the discount during the selling period. If the retailer is capable and expert instead, i.e. $\delta$ lies above the threshold, then the best policy is first to increase the discount and, in case, to decrease it at the end of the selling period. This means that a skilful retailer, if properly motivated at the beginning of the sales period, may provide an appropriate sales level even when discounts become lower. With unskilled retailers it is always necessary to increase the discount to obtain a higher profit reached through a price policy since "service" is not good enough. Formally, the optimal policy is given in the following Proposition, the proof of which is reported in Appendix B.

Proposition 3.1. Let be $\delta_{0}=\theta \eta /(\eta \gamma+\epsilon)$.

Case 1: The inefficient retailer.

If the retailer's efficiency is low, i.e. $\delta \leqslant \delta_{0}$, then the optimal trade discount policy is a continuous increasing function given by

$$
\alpha^{*}(t)= \begin{cases}\alpha_{1} & t \in\left[t_{1}, \tau_{1}\right), \\ D_{1} \mathrm{e}^{\lambda t}+D_{2} \mathrm{e}^{-\lambda t}+\left(\bar{\alpha}+\alpha_{2}\right) / 2 & t \in\left[\tau_{1}, t_{2}\right],\end{cases}
$$

where $\tau_{1} \in\left[t_{1}, t_{2}\right], D_{1} \geqslant 0$ and $D_{2}<0$ are constants and $\lambda=\sqrt{(\theta-\gamma \delta) \delta \epsilon / \eta}$.

Case 2: The efficient retailer.

If the retailer's efficiency is high, i.e. $\delta>\delta_{0}$, then the optimal trade discount policy is a continuous quasi-concave function

$$
\alpha^{*}(t)= \begin{cases}\alpha_{1} & t \in\left[t_{1}, \tau_{1}\right), \\ D_{1} \mathrm{e}^{\lambda t}+D_{2} \mathrm{e}^{-\lambda t}+\left(\bar{\alpha}+\alpha_{2}\right) / 2 & t \in\left[\tau_{1}, \tau_{2}\right], \\ \alpha_{1} & t \in\left(\tau_{2}, t_{2}\right],\end{cases}
$$

where $t_{1} \leqslant \tau_{1} \leqslant \tau_{2} \leqslant t_{2}, D_{1}<0$ and $D_{2}<0$ are constants. ${ }^{2}$ If $\tau_{1}=t_{1}$ then $\alpha^{*}\left(\tau_{1}\right) \leqslant \alpha^{*}\left(\tau_{2}\right){ }^{3}$

\footnotetext{
${ }^{2}$ The way to compute $D_{1}, D_{2}, \tau_{1}$ and $\tau_{2}$ is rather technical and explained in Appendix B.4 (in particular see the Proof of Lemma B.3).

${ }^{3}$ Due to the quasi-concavity of the optimal trade discount, inequality $\alpha^{*}\left(\tau_{1}\right) \leqslant \alpha^{*}\left(\tau_{2}\right)$ means that even if the optimal discount starts at a level $\alpha^{*}\left(\tau_{1}\right)$ greater than $\alpha_{1}$ (this can only happen when $\tau_{1}=t_{1}$ ) then it must any first increase and then, at the end of the selling period, it can decrease but, in any case, its value $\alpha^{*}\left(\tau_{2}\right)$ cannot reach values lower than the initial one.
} 
Optimal sales level $x^{*}(t)$ and optimal retailer motivation $M^{*}(t)$ can be calculated by substituting $\alpha^{*}(t)$ in the motion equations of Problem $P$.

The rationale behind the above Proposition is the following. As soon as the selling skill becomes too low the positive effect of the retailer's motivation on sales is lowered. To keep an adequate profit level, it becomes necessary to provide an always increasing wholesale price discount. This action will compensate the unsatisfactory behavior of the retailer, who is unable to contrast the saturation effect of the market. On the other hand, if the retailer is rather efficient, it is sufficient to push the product via trade discounts at the beginning of the selling period, since this turns out to increase the motivation and the performance of the retailer. This way the good performance of the retailer may allow to decrease the wholesale price discount at the end of the selling period.

We provide now two examples which exhibit the two possible optimal trade discount policies described in the above Proposition. In the first example an inefficient retailer $\left(\delta \leqslant \delta_{0}\right)$ requires an increasing trade discount while in the second one an efficient retailer $\left(\delta>\delta_{0}\right)$ allows the manufacturer to offer a lower trade discount at the end of the selling period.

Example 1. Let us consider problem $P$ in the time period starting at $t_{1}=0$ and ending at $t_{2}=2$, with the following values of the parameters: $p=2.9, c_{0}=0.8, \beta=0.1215, \gamma=0.7(1-\beta) \approx 0.615, \epsilon=0.6(1-\beta) \approx 0.527, \bar{\alpha}=0.25, \alpha_{1}=0.2, \alpha_{2}=$ $\left(p-c_{0}\right) / p \approx 0.7241, \delta=0.8, \tilde{\eta}=0.8, \theta=10, \bar{M}=5$.

We have an inefficient retailer (or a relatively small market), i.e. Case 1 of Proposition 3.1. The optimal wholesale price discount must be positive and strictly increasing in $\left[\tau_{1}, t_{2}\right]$ to guarantee the maximum profit of the manufacturer. In fact, at the beginning of the sale period the optimal policy is to keep the trade discount at its lowest level $\alpha_{1}$ (Fig. 1a). During the same period cumulative sales and motivation increase steeply (Figs. $1 \mathrm{~b}$ and c), then saturation effect becomes relevant and the growth of motivation stops for a while. To reach a higher market share it is necessary (from time $\tau_{1} \approx 0.50977$ ) to increase the wholesale price discount. This has a positive effect on motivation, which starts to grow again, and also on sales: the cumulative sales increase, but slower due to the strong saturation effect.

Example 2. Let us consider problem $P$ with the same values of the parameters as in Example 1 except for: $t_{2}=4, \beta=0.15, \gamma=0.7(1-\beta) \approx 0.595, \epsilon=0.6(1-\beta) \approx 0.510, \theta=1$, and $\bar{M}=0.5$.

With respect to Example 1 the main change concerns parameter $\theta$ and the initial motivation $\bar{M}$, some slight variations of other parameters are only useful to receive nice pictures. Now we have essentially the same retailer as before $(\delta$ is the same) but he sells in a very large market ( $\theta$ is now one tenth as in Example 1) so it is much easier for the retailer to sell. The retailer is considered efficient with respect to this market, we have Case 2 of Proposition 3.1. The optimal choice is to allow increasing wholesale price discounts starting at $\tau_{1} \approx 0.777$ and to reduce them at the end of the sale period, reaching the wholesale list price at time $\tau_{2} \approx 3.783$ (Fig. 2a). At the beginning of the selling period cumulative sales increase rapidly (Fig. 2b) but the retailer's motivation needs to be supported by trade discounts since its growth is decreasing before $\tau_{1}$ (Fig. 2c). The width of the market allows to reduce wholesale price discounts in the second part of the selling period, since the saturation effect is rather weak and at the same time motivation has raised sufficiently high levels.

Observe that the switching times $\tau_{1}$ and $\tau_{2}$ may coincide with $t_{1}$ or $t_{2}$. In that case the optimal policies can be thought, qualitatively, as "a part" of those described in the above instances. For example suppose that, with an efficient retailer, one obtains $\tau_{1}>t_{1}$ and $\tau_{2}=t_{2}$ then, qualitatively, the graph of the optimal discount policy would be similar to that of the first two tracts of Fig. 2a, first a constant discount $\alpha_{1}$ then a strictly concave discount till $t_{2}$.

We can also observe that, as a by-product of the above Proposition we also have that the maximum discount policy, i.e. discount equal to $\alpha_{2}$ on the whole time window, cannot be optimal.
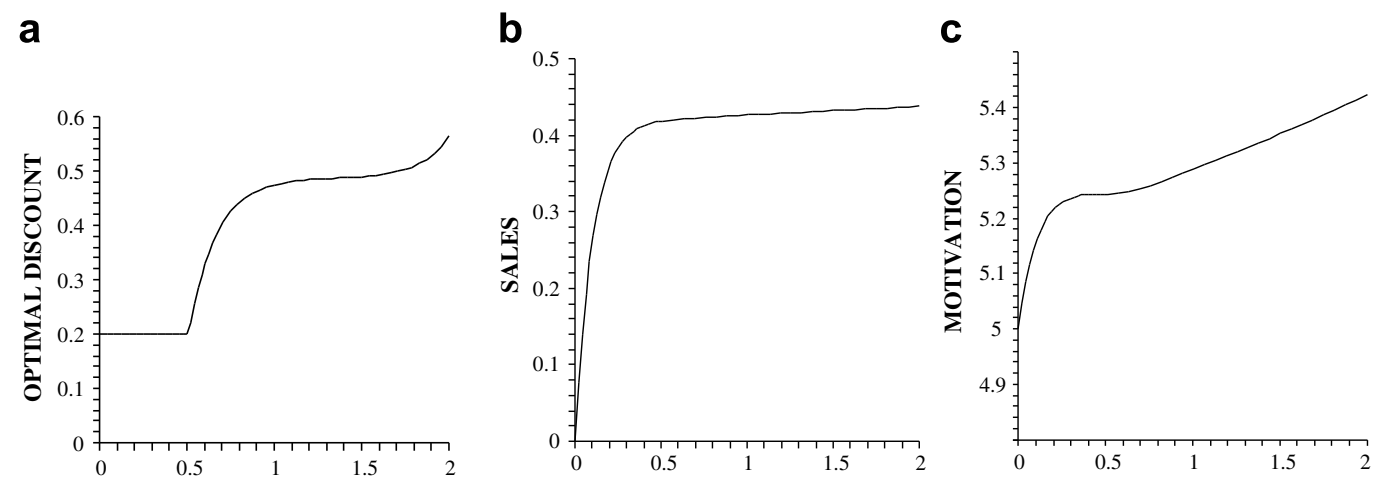

Fig. 1. Optimal discount policy (a), optimal sales path (b) and optimal motivation path (c) for Example 1. 

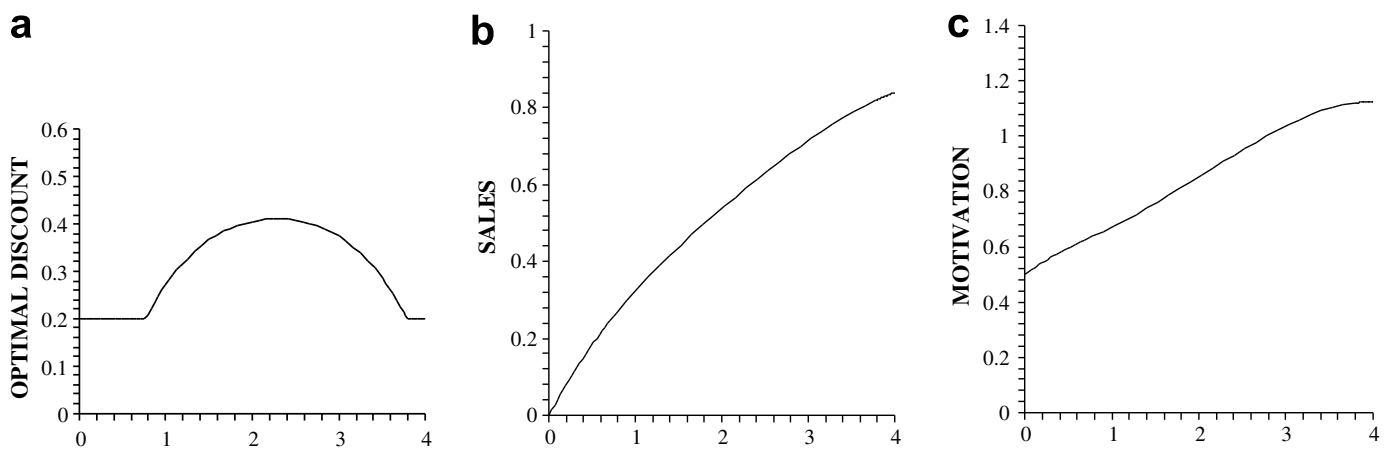

Fig. 2. Optimal discount policy (a), optimal sales path (b) and optimal motivation path (c) for Example 2.

It is interesting to reread the above proposition in terms of market width, even if it is not the point of view we want to take into consideration here. Observe that inequality $\delta \leqslant \theta \eta /(\eta \gamma+\epsilon)$, which characterizes the inefficient retailer, can be rewritten as $\theta \geqslant \delta(\gamma+\epsilon / \eta)$. This means that with small markets ( $\theta$ high) it is convenient to act as with inefficient retailers, an aggressive increase of the discount during the selling period is the best choice. Similarly, large markets allow to "relax" at the end of the period. In other words the retailer can be considered good or not with respect to the width of the market, e.g. he is inefficient if $\delta / \theta \leqslant \eta /(\eta \gamma+\epsilon)$.

\section{Profit sensitivity to retailer's skill and pass-through}

Let us consider now in more detail the case of a (relatively) inefficient retailer, which is the critical case for the manufacturer. We will shortly analyze how the optimal profit value of problem $P$ can be changed acting on the parameters of the model which are more closely related to the retailer's features and choices. More precisely the parameters which will be considered are the retailer's efficiency $\delta$ and the pass-through $\beta$. In our model these parameters are considered exogenously fixed. Nevertheless we observe that changes in the retailer's efficiency can be obtained by vocational training aimed at improving his professional competence, his knowledge on the product and so on. Likewise, also pass-through can be negotiated, but to allow a higher pass-through a retailer will probably require some, may be non-monetary, compensation. In any case, improving the efficiency of the retailer or to increase the pass-through will usually be expensive and compelling for the manufacturer. Therefore it becomes rather interesting to analyze the effects of changes in $\delta$ or $\beta$ on her profit, we do this by means of some numerical experiments.

The first computation we present concerns the evaluation of the profit in 2500 instances of the optimal control problem $P$ obtained by varying the retailer's efficiency $\delta$ and the pass-through $\beta$ decided by the retailer. To restrict the analysis to the inefficient retailer case we consider $\delta \in[1.00,1.15]$ and $\beta \in[0.70,0.90]$. The other parameters of the model, which are related to the market features, are as follows: $t_{1}=0, t_{2}=2, p=2, c_{0}=1, \theta=0.3, \tilde{\eta}=4, \gamma=0.3(1-\beta), \epsilon=$ $1-\beta, \bar{\alpha}=0.4, \alpha_{1}=0.1, \alpha_{2}=\left(p-c_{0}\right) / p=0.5, \bar{M}=2$.

Computations show that:

- For high values of efficiency $\delta$ manufacturer's profit decreases as the pass-through $\beta$ increases.

- For low values of $\delta$ manufacturer's profit increases with respect to $\beta$ (see Fig. 3).

The result is not surprising. In fact, a low pass-through will increase a good vendor's motivation and his ability to sell is sufficient to overcome the sales reduction effect due to the lower discount transmitted to the consumers. If the efficiency of the retailer is very low, instead, the manufacturer profit takes higher advantage from the sales increase obtained selling at a reduced price due to a higher pass-through. The retailer in fact, even if properly motivated, will not provide a high growth of sales.

It is interesting to remark that

- for intermediate values of efficiency $\delta$, the manufacturer profit is non monotone.

In this case the maximum profit with respect to pass-through $\beta$ is obtained in an internal point of the domain $[0.70,0.90]$ (see Fig. 4). In that point the vendor's motivation and ability balance the low pass-through effect on final price. Our numerical simulations suggest in any case that this kind of behavior of the profit function seems to be relatively "rare". 


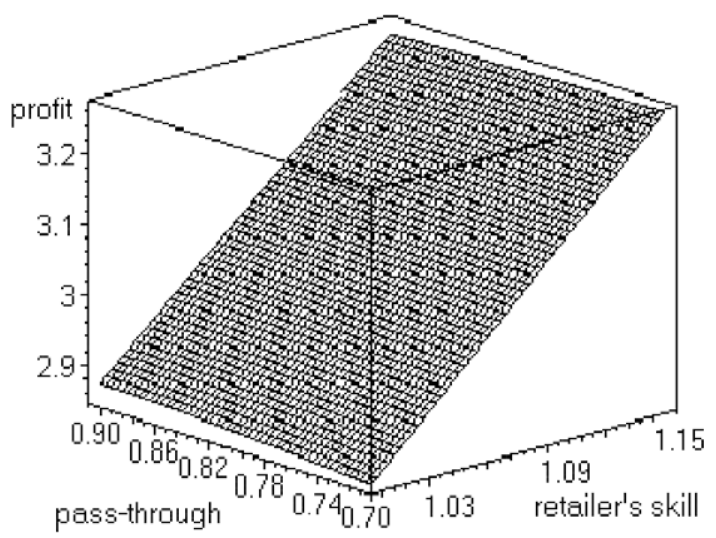

Fig. 3. Maximum profit for $\delta \in[1.00,1.15]$ and $\beta \in[0.70,0.90]$.

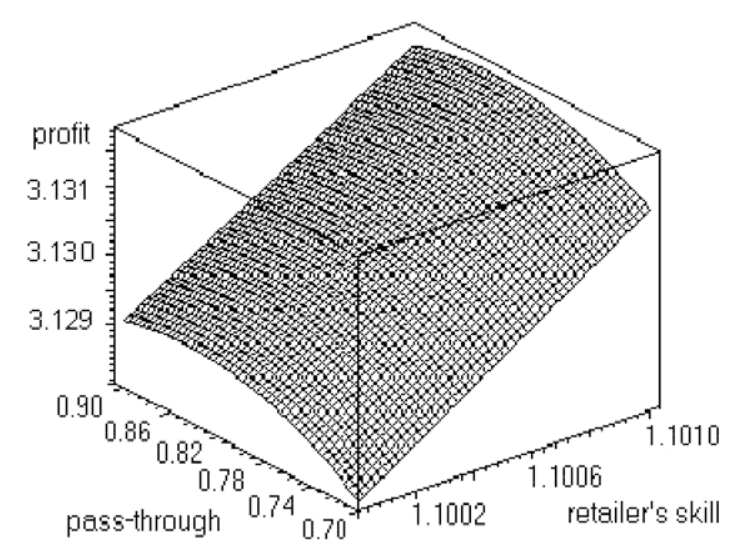

Fig. 4. Maximum profit for $\delta \in[1.100,1.101]$ and $\beta \in[0.70,0.90]$.

\section{Conclusion}

The primary purpose of this research was to enhance our understanding on the manufacturer-retailer relationship by means of a model in which the role of sale motivation and skill of the retailer are explicitly considered. We propose a dynamic optimization model where the retailer is motivated in his selling activity, according to manufacturer's benefits.

The qualitative properties of the solutions of the model are obtained by means of standard optimal control tools and have several notable marketing implications.

First, we show that the optimal discount policy of the manufacturer in a vertical channel depends on the efficiency of the retailer. In particular an increasing discount policy is convenient for inefficient retailers while efficient retailers may allow the manufacturer to decrease trade discounts at the end of the selling period. We observed that efficiency of the retailer strongly depends on the dimension of the market. In particular in a rather large market it is much easier for a retailer to be considered efficient. The interesting relationship between retailer's efficiency and market dimension will be further investigated in future research.

A second result is obtained through numerical experiments. We show that pass-through strongly influences the value of the optimal profit: in particular a higher pass-through produces higher profit if the retailer is very inefficient but lower profit if the retailer is not overly inefficient, his skill and motivation compensate in this case the negative effect on sales of a high final price.

Both these results imply that from the manufacturer's point of view it is necessary to develop a monitoring program to distinguish between the retailer's intrinsic capabilities, his skill and qualification, and his sale motivation, his zeal and engagement.

Managerially, the monotonicity or quasi-concavity properties of the optimal discount also suggests that piecewise constant trade discounts (to be used in practice) maintaining the same type of monotonicity could be a good approximation of the optimal behavior. Moreover it is also essential for manufacturers' profit maximization to consider different ways of improving retailers' performances in order to deal exclusively with efficient retailers. For example, Basuroy et al. (2001) 
study the impact of category management on retailer performance, a management initiative that aims to improve retailer performances through more coordinate buying, merchandizing and pricing.

A possible way to consider conflict and coordination is via a game theoretic approach (see e.g. Jørgensen and Zaccour, 2004). For example it is possible to consider the Stackelberg problem in which the retailer chooses the pass-through to maximize his profit or by analyzing the case of channel coordination between manufacturer and retailer in a differential game framework.

Another possible generalization of the model, which could also be the subject of future research, is to consider a multisegment market (see e.g. Buratto et al., 2006) with several retailers and with constraint on final motivations and sales levels.

Besanko et al. (2005) show that pass-through varies substantially across products and categories, that own-brand passthrough rates are, on average, quite large and that cross-brand pass-through is positively or negatively significant. These results suggest another useful extension of our model: from a single good to a multiple goods model.

\section{Acknowledgements}

Supported by Università Ca' Foscari di Venezia, MIUR - PRIN co-financing 2005, and the Council for Grants (under RF President) and State Aid of Fundamental Science Schools (Grant No. NSh-4999.2006.6). We would like to thank Bruno Viscolani and the anonymous referees for constructive suggestions and comments.

\section{Appendix A. Concavity of the cumulative sales function}

Let us prove that (2) is a necessary and sufficient condition to have a concave cumulative sales function $x(t)$ for every constant wholesale price discount. In the following we will set

$$
a=\theta-\gamma \delta, \quad b=a \eta-\delta \epsilon=(\theta-\gamma \delta) \eta-\delta \epsilon .
$$

Observe that if $a=0$ it is not possible to obtain concavity of $x(t)$ for every constant trade discount $\alpha(t)$ since if, for example, $\alpha(t)=\alpha_{2}$ then $\ddot{x}(t)>0$.

Consider the case $a \neq 0$. Setting $\alpha(t)=\hat{\alpha}$ in the system of differential equations of problem $P$ one has

$$
x(t)=C(\hat{\alpha})\left[1-\mathrm{e}^{a\left(t_{1}-t\right)}\right]+\delta \epsilon(\hat{\alpha}-\bar{\alpha})\left(t-t_{1}\right) / a,
$$

where $C(\hat{\alpha})=(a \delta \bar{M}+b \hat{\alpha}+\delta \epsilon \bar{\alpha}) / a^{2}=(a(\delta \bar{M}+\eta \hat{\alpha})+\delta \epsilon(\bar{\alpha}-\hat{\alpha})) / a^{2}$. So the cumulative sales function is concave for every $\hat{\alpha} \in\left[\alpha_{1}, \alpha_{2}\right]$ if and only if $C(\hat{\alpha}) \geqslant 0 \forall \hat{\alpha} \in\left[\alpha_{1}, \alpha_{2}\right]$, i.e. if and only if

$$
a \delta \bar{M}+b \hat{\alpha}+\delta \epsilon \bar{\alpha} \geqslant 0 \forall \hat{\alpha} \in\left[\alpha_{1}, \alpha_{2}\right] \text {, }
$$

i.e.

$$
\theta \geqslant\left((\epsilon(\hat{\alpha}-\bar{\alpha})) /\left(\delta \bar{M}+\eta \alpha_{2}\right)+\gamma\right) \delta \quad \forall \hat{\alpha} \in\left[\alpha_{1}, \alpha_{2}\right],
$$

which is true if and only if (2) holds. Remark that assumption (2) implies $a=\theta-\gamma \delta>0$.

\section{Appendix B. Solution of problem $P$}

\section{B.1. An equivalent problem}

Let us first rewrite problem $P$ in an equivalent problem which can be solved easier.

Let us consider the new control variable $u(t)=\alpha(t)-\alpha_{2}$, the new state variable $y(t)=-\theta x(t)+\delta M(t)+\alpha_{2} \eta$ and denote

$$
u_{1}=\alpha_{1}-\alpha_{2}<0, \quad \bar{u}=\bar{\alpha}-\alpha_{2}<0, \quad y_{1}=\delta \bar{M}+\alpha_{2} \eta .
$$

Now we can write our model in the following equivalent form:

$$
\begin{array}{cl}
Q: \text { maximize } & -p \int_{t_{1}}^{t_{2}}[y(t)+\eta u(t)] u(t) \mathrm{d} t, \\
\text { subject to } & \dot{x}(t)=y(t)+\eta u(t), \\
& \dot{y}(t)=-a y(t)-b u(t)-c, \\
& x\left(t_{1}\right)=0, \quad y\left(t_{1}\right)=y_{1}, \\
& u(t) \in\left[u_{1}, 0\right],
\end{array}
$$

where $a=\theta-\delta \gamma>0, b=a \eta-\delta \epsilon$ and $c=\delta \epsilon \bar{u}<0$.

Remark that $u(t) \leqslant 0 \forall t$ and, since $\alpha_{1} \leqslant \bar{\alpha}$ and $\alpha_{1}<\alpha_{2}$, 
$2 u_{1}<\bar{u}$

Moreover, assumption (2) can be rewritten as

$a y_{1}+c \geqslant 0$.

Problem $Q$ allows to write $\dot{x}(t)=\left(y_{1}+\frac{c}{a}\right) \mathrm{e}^{a\left(t_{1}-t\right)}-b \mathrm{e}^{-a t} \int_{t_{1}}^{t} u(\tau) \mathrm{e}^{a \tau} \mathrm{d} \tau-\frac{c}{a}+\eta u(t)$.

Since we require, obviously, non negative sales intensity this could require, for negative values of $b$, a "sufficiently short" sales period $\left[t_{1}, t_{2}\right]$.

\section{B.2. Hamiltonian and maximum principle}

Given problem $Q$ consider its Hamiltonian function

$$
H=-p[y(t)+\eta u(t)] u(t)+z_{0}(t)[y(t)+\eta u(t)]+z(t)[-a y(t)-b u(t)-c]
$$

and the Lagrangian function

$$
L=H+\mu_{1}(t)\left[u(t)-u_{1}\right]-\mu_{2}(t) u(t) .
$$

Due to the Pontryagin Maximum Principle (in particular, see (Sethi and Thompson, 2000, p. 61)) one has that if $u^{*}(t)$ is the optimal control and $x^{*}(t), y^{*}(t)$ are the optimal state variables, then continuous and piecewise continuously differentiable functions (adjoint functions) $z_{0}(t)$ and $z(t)$ and piecewise continuous functions (Lagrange multipliers) $\mu_{1}(t)$ and $\mu_{2}(t)$ must exist such that

$$
\begin{aligned}
& \dot{z}_{0}(t)=-\frac{\partial L^{*}}{\partial x} \quad \dot{z}(t)=-\frac{\partial L^{*}}{\partial y} \quad z_{0}\left(t_{2}\right)=0 \quad z\left(t_{2}\right)=0 \quad \frac{\partial L^{*}}{\partial u}=0 \\
& \mu_{1}(t) \geqslant 0, \quad \mu_{1}(t)\left[u^{*}(t)-u_{1}\right]=0, \quad \mu_{2}(t) \geqslant 0, \quad \mu_{2}(t) u^{*}(t)=0,
\end{aligned}
$$

where $L^{*}=L\left(y^{*}, x^{*}, u^{*}, z, z_{0}, \mu_{1}, \mu_{2}\right)$.

With the aim to find the structure of the optimal control of problem $P$ we first state some preliminary propositions.

Proposition B.1. Functions $\mu_{1}(t)$ and $\mu_{2}(t)$ are continuous.

Proof. Since in our case $\partial L^{*} / \partial x \equiv 0$ then $z_{0}(t) \equiv 0$ and

$$
\dot{z}(t)=-\frac{\partial L^{*}}{\partial y}=a z(t)+p u^{*}(t) .
$$

Moreover (see (12)) from $\partial L^{*} / \partial u=0$ one has

$$
\mu_{1}(t)-\mu_{2}(t)=p y^{*}(t)+b z(t)+2 p \eta u^{*}(t) .
$$

Function $u^{*}(t)$ is continuous since the Hamiltonian function $H$ is strictly concave with respect to $u$ (see e.g. (Seierstad and Sydsæter, 1987, p. 86, Note 2(b))), so also $\mu_{1}(t)-\mu_{2}(t)$ is continuous. From the complementary conditions (14) we have $\mu_{1}(t) u^{*}(t)-\mu_{1}(t) u_{1}-\mu_{2}(t) u^{*}(t)=0$ therefore $\mu_{1}(t) u_{1}=\left(\mu_{1}(t)-\mu_{2}(t)\right) u^{*}(t)$ it follows that both $\mu_{1}$ and $\mu_{2}$ are continuous.

Proposition B.2. (a) $\mu_{2}(t)=0 \forall t \in\left[t_{1}, t_{2}\right]$; (b) if $\mu_{1}\left(\rho_{1}\right)=\mu_{1}\left(\rho_{2}\right)=0$ then $\mu_{1}(t)=0 \forall t \in\left[\rho_{1}, \rho_{2}\right]$.

Proof. (a) Suppose that $\mu_{2}(\hat{t})>0$ for some $\hat{t} \in\left(t_{1}, t_{2}\right)$ then, due to the continuity of $\mu_{2}$, some interval exists in which $\mu_{2}$ keeps strictly positive: let $I=\left(\rho_{1}, \rho_{2}\right) \subset\left[t_{1}, t_{2}\right]$ be the interval of maximum length such that $\mu_{2}(t)>0 \forall t \in I$. By complementary conditions (14) this implies $u^{*}(t)=\mu_{1}(t)=0 \forall t \in\left(\rho_{1}, \rho_{2}\right)$. Hence in $\left(\rho_{1}, \rho_{2}\right)$ (16) becomes

$$
\mu_{2}(t)=-p y^{*}(t)-b z(t)
$$

where $y^{*}(t)$ and $z(t)$ satisfy the differential equations $\dot{y}(t)=-a y(t)-c \dot{z}(t)=a z(t)$ (see definition of problem $Q$ and (15)), i.e.

$$
y^{*}(t)=C_{y} \mathrm{e}^{-a t}-c / a \quad z(t)=C_{z} \mathrm{e}^{a t}
$$

and $C_{y}$ and $C_{z}$ are given by

$$
C_{y}=\left[y^{*}\left(\rho_{1}\right)+c / a\right] \mathrm{e}^{a \rho_{1}}, \quad C_{z}=z\left(\rho_{2}\right) \mathrm{e}^{-a \rho_{2}} .
$$

Moreover, $\ddot{\mu}_{2}(t)=a^{2}\left(-p C_{y} \mathrm{e}^{-a t}-b C_{z} \mathrm{e}^{a t}\right)=a^{2}\left[\mu_{2}(t)-p c / a\right]$. So $\ddot{\mu}_{2}(t)>0$ since $\mu_{2}(t)>0$ and $c<0$. Hence $\mu_{2}(t)$ is convex in $\left[\rho_{1}, \rho_{2}\right]$.

Consider now the cases (i) $\rho_{1}=t_{1}, \rho_{2}=t_{2}$, (ii) $\rho_{1}=t_{1}, \rho_{2}<t_{2}$, (iii) $\rho_{1}>t_{1}, \rho_{2}=t_{2}$, (iv) $\rho_{1}>t_{1}, \rho_{2}<t_{2}$. 
(i) Using the boundary conditions $y\left(t_{1}\right)=y_{1}, z\left(t_{2}\right)=0$ one has $\mu_{2}(t)=p\left\{-y_{1} \mathrm{e}^{a\left(t_{1}-t\right)}+c\left[1-\mathrm{e}^{a\left(t_{1}-t\right)}\right] / a\right\} \forall t \in\left[t_{1}, t_{2}\right]$. Therefore, since $c<0$, one has $\mu_{2}(t)<0$ in the whole interval, which contradicts complementary conditions.

(ii) Using the initial condition $y\left(t_{1}\right)=y_{1}$, one has $y^{*}(t)=C_{y} \mathrm{e}^{-a t}-c / a \geqslant 0$ therefore, in order to keep $\mu_{2}(t)>0$, we need $-b z(t)>0$. This way, recalling that $a y_{1}+c \geqslant 0$, we have $\dot{\mu}_{2}(t)=-p \dot{y}^{*}-b \dot{z}=p\left(a y_{1}+c\right) \mathrm{e}^{a\left(t_{1}-t\right)}-b a z(t)>$ $0 \forall t \in\left[t_{1}, \rho_{2}\right]:$ again we have a contradiction since $\mu_{2}\left(t_{1}\right)>0$ and $\mu_{2}\left(\rho_{2}\right)=0$.

(iii) Using the final condition $z\left(t_{2}\right)=0$, one has $\mu_{2}(t)=-p y^{*}(t)$; therefore $\mu_{2}(t)>0$ if and only if $y^{*}(t)<0$. But $y^{*}(t)=C_{y} \mathrm{e}^{-a t}-c / a<0$ requires $C_{y}<0$, therefore $\dot{\mu}_{2}(t)=-p \dot{y}^{*}=a p C_{y} \mathrm{e}^{-a t}<0$. We have a contradiction since $\mu_{2}\left(\rho_{1}\right)=0$ and $\mu_{2}\left(t_{2}\right)>0$.

(iv) In this case $\mu_{2}\left(\rho_{1}\right)=\mu_{2}\left(\rho_{2}\right)=0$ thus, by convexity of $\mu_{2}, \mu_{2}(t)=0$ on the whole interval $\left(\rho_{1}, \rho_{2}\right)$, again a contradiction.

Therefore $\mu_{2}$ can never be strictly positive.

(b) The proof is quite similar to the proof of case (iv). Indeed, using (10) it is possible to show that function $\mu_{1}$ is convex in $\left(\rho_{1}, \rho_{2}\right)$.

\section{B.3. The proof of Proposition 3.1}

We split the proof by means of some lemmas.

Lemma B.1. If $\mu_{1}(t)=0, \forall t \in\left[\rho_{1}, \rho_{2}\right] \subseteq\left[t_{1}, t_{2}\right]$ then in $\left[\rho_{1}, \rho_{2}\right]$ optimal control $u^{*}(t)$, optimal state variable $y^{*}(t)$ and adjoint function $z(t)$ are

$$
\begin{aligned}
& u^{*}(t)=D_{1} \mathrm{e}^{\lambda t}+D_{2} \mathrm{e}^{-\lambda t}+\bar{u} / 2, \\
& y^{*}(t)=\frac{a b^{2}}{\eta(a+\lambda)^{2}} C_{1} \mathrm{e}^{\lambda t}+\frac{\eta(a+\lambda)^{2}}{a} C_{2} \mathrm{e}^{-\lambda t}-\frac{b \bar{u}+2 c}{2 a}, \\
& z(t)=p\left[C_{1} \mathrm{e}^{\lambda t}+C_{2} \mathrm{e}^{-\lambda t}-\bar{u} /(2 a)\right],
\end{aligned}
$$

where $\lambda=\sqrt{(a \delta \epsilon) / \eta}, C_{1}, C_{2}$ are some constants and

$$
D_{1}=(\lambda-a) C_{1}, \quad D_{2}=-(\lambda+a) C_{2} .
$$

Proof. Due to (16), Proposition B.2 and the hypothesis, one has

$$
u^{*}(t)=-[p y(t)+b z(t)] /(2 p \eta), t \in\left[\rho_{1}, \rho_{2}\right] .
$$

Substituting this expression into the motion equation of $y(t)$ and the adjoint motion equation of $z(t)$ (see definition of problem $Q$ and (15)) and solving the system

$$
\left\{\begin{array}{l}
\dot{y}(t)=-a y(t)-b u(t)-c \\
\dot{z}(t)=a z(t)+p u(t)
\end{array}\right.
$$

we obtain its general solution which can be written as (21), (22). Expression (20) can be obtained now from (24).

Lemma B.2. Optimal control $u^{*}(t)$, optimal state variable $y^{*}(t)$ and adjoint function $z(t)$ of problem $Q$ are:

$$
\begin{aligned}
& u^{*}(t)= \begin{cases}u_{1}, & t \in\left[t_{1}, \tau_{1}\right), \\
D_{1} \mathrm{e}^{\lambda t}+D_{2} \mathrm{e}^{-\lambda t}+\bar{u} / 2, & t \in\left[\tau_{1}, \tau_{2}\right], \\
u_{1}, & t \in\left(\tau_{2}, t_{2}\right],\end{cases} \\
& y^{*}(t)= \begin{cases}\left(y_{1}+\frac{b u_{1}+c}{a}\right) \mathrm{e}^{a\left(t_{1}-t\right)}-\frac{b u_{1}+c}{a}, & t \in\left[t_{1}, \tau_{1}\right), \\
\frac{a b^{2}}{\eta(a+\lambda)^{2}} C_{1} \mathrm{e}^{\lambda t}+\frac{\eta(a+\lambda)^{2}}{a} C_{2} \mathrm{e}^{-\lambda t}-\frac{b \bar{u}+2 c}{2 a}, & t \in\left[\tau_{1}, \tau_{2}\right], \\
C_{y} \mathrm{e}^{-a t}-\frac{b u_{1}+c}{a}, & t \in\left(\tau_{2}, t_{2}\right],\end{cases} \\
& z(t)= \begin{cases}C_{z} \mathrm{e}^{a t}-p u_{1} / a, & t \in\left[t_{1}, \tau_{1}\right), \\
p\left[C_{1} \mathrm{e}^{\lambda t}+C_{2} \mathrm{e}^{-\lambda t}-\bar{u} /(2 a)\right], & t \in\left[\tau_{1}, \tau_{2}\right], \\
p u_{1}\left[\mathrm{e}^{a\left(t-t_{2}\right)}-1\right] / a, & t \in\left(\tau_{2}, t_{2}\right],\end{cases}
\end{aligned}
$$

where $t_{1} \leqslant \tau_{1} \leqslant \tau_{2} \leqslant t_{2}, C_{1}, C_{2}, C_{y}, C_{z}$ are some constants, $D_{1}, D_{2}$ are given by (23). 
Proof. Let us first prove that it is not possible to find $\rho_{1}, \rho_{2} \in\left[t_{1}, t_{2}\right]$ such that $\rho_{1}<\rho_{2}$ and $u^{*}(t)=0 \forall t \in\left[\rho_{1}, \rho_{2}\right]$. Indeed, suppose by contradiction that $u^{*}(t)=0 \forall t \in\left[\rho_{1}, \rho_{2}\right]$; then by complementary conditions (14) it must be $\mu_{1}(t)=0 \forall t \in\left[\rho_{1}, \rho_{2}\right]$. Therefore, using Lemma B.1, the optimal control $u^{*}(t)$ is given by (20) in the whole interval $\left[\rho_{1}, \rho_{2}\right]$. This implies that $u^{*}(t)=0 \forall t \in\left[\rho_{1}, \rho_{2}\right]$ if and only if $D_{1}=D_{2}=0$ and $\bar{u}=0$, which is impossible since $\bar{u}<0$ (see (9)). Therefore $u^{*}(t)$ cannot be identically equal to zero in the whole interval $\left[\rho_{1}, \rho_{2}\right]$. This means that $u^{*}(t)$ can either be given by (20) or be equal to $u_{1}$. Due to Proposition B.2 the only possible optimal control form is therefore given by (25).

Let us remark that the general solution of the system

$$
\dot{y}(t)=-a y(t)-b u_{1}-c, \quad \dot{z}(t)=a z(t)+p u_{1}
$$

is $y(t)=C_{y} \mathrm{e}^{-a t}-\left(b u_{1}+c\right) / a, z(t)=C_{z} \mathrm{e}^{a t}-p u_{1} / a$, where $C_{y}$ and $C_{z}$ are some arbitrary constant. Using (25), Lemma B.1 (see (21) and (22)), boundary conditions $y\left(t_{1}\right)=y_{1}$ and $z\left(t_{2}\right)=0$, and continuity of $u^{*}(t), y^{*}(t)$ and $z(t)$, we obtain (26) and (27).

\section{Lemma B.3}

$$
D_{2}<0, \quad \operatorname{sign} D_{1}=\operatorname{sign} b .
$$

Proof. Using (25)-(27) and the continuity of $u^{*}(t), y^{*}(t), z(t)$ one has

$$
\begin{aligned}
& \frac{a b^{2}}{\eta(a+\lambda)^{2}} C_{1} \mathrm{e}^{\lambda \tau_{1}}+\frac{\eta(a+\lambda)^{2}}{a} C_{2} \mathrm{e}^{-\lambda \tau_{1}}-\frac{b \bar{u}+2 c}{2 a}=y^{*}\left(\tau_{1}\right), \\
& {\left[D_{1} \mathrm{e}^{\lambda \tau_{1}}+D_{2} \mathrm{e}^{-\lambda \tau_{1}}+\bar{u} / 2\right]\left(\tau_{1}-t_{1}\right)=u_{1}\left(\tau_{1}-t_{1}\right),} \\
& p\left[C_{1} \mathrm{e}^{\lambda \tau_{2}}+C_{2} \mathrm{e}^{-\lambda \tau_{2}}-\bar{u} /(2 a)\right]=z\left(\tau_{2}\right), \\
& {\left[D_{1} \mathrm{e}^{\lambda \tau_{2}}+D_{2} \mathrm{e}^{-\lambda \tau_{2}}+\bar{u} / 2\right]\left(\tau_{2}-t_{2}\right)=u_{1}\left(\tau_{2}-t_{2}\right) .}
\end{aligned}
$$

Let us look now how to express coefficients $D_{1}$ and $D_{2}$ depending on the values of $\tau_{1}$ and $\tau_{2}$. Using (30) and (32) we get

$$
\tau_{1} \neq t_{1} \quad \tau_{2} \neq t_{2} \Rightarrow D_{1}=\frac{u_{1}-\bar{u} / 2}{\mathrm{e}^{\lambda \tau_{1}}+\mathrm{e}^{\lambda \tau_{2}}} \quad D_{2}=D_{1} \mathrm{e}^{\lambda\left(\tau_{1}+\tau_{2}\right)} .
$$

By means of (29) and (30) we obtain

$$
\tau_{1} \neq t_{1} \Rightarrow\left\{\begin{array}{l}
D_{1}=\frac{1}{2 \eta \lambda}\left[\left(a y_{1}+b u_{1}+c\right) \mathrm{e}^{a\left(t_{1}-\tau_{1}\right)}+(\eta \lambda+\delta \epsilon)\left(u_{1}-\frac{\bar{u}}{2}\right)\right] \mathrm{e}^{-\lambda \tau_{1}}, \\
D_{2}=\frac{1}{2 \eta \lambda}\left[-\left(a y_{1}+b u_{1}+c\right) \mathrm{e}^{a\left(t_{1}-\tau_{1}\right)}+(\eta \lambda-\delta \epsilon)\left(u_{1}-\frac{\bar{u}}{2}\right)\right] \mathrm{e}^{\lambda \tau_{1}}, \\
\dot{u}^{*}\left(\tau_{1}+0\right)=\frac{1}{\eta}\left[\left(a y_{1}+b u_{1}+c\right) \mathrm{e}^{a\left(t_{1}-\tau_{1}\right)}+\delta \epsilon\left(u_{1}-\frac{\bar{u}}{2}\right)\right]>0,
\end{array}\right.
$$

where $\dot{u}^{*}\left(\tau_{1}+0\right)$ denotes the right derivative of $\dot{u}^{*}(t)$ at $\tau_{1}$.

From (31), (32), (9) and (10) one has

$$
\tau_{2} \neq t_{2} \Rightarrow\left\{\begin{array}{l}
D_{1}=\frac{1}{2 \eta \lambda}\left[-b u_{1} \mathrm{e}^{a\left(\tau_{2}-t_{2}\right)}+(\eta \lambda-\delta \epsilon)\left(u_{1}-\frac{\bar{u}}{2}\right)\right] \mathrm{e}^{-\lambda \tau_{2}}, \\
D_{2}=\frac{1}{2 \eta \lambda}\left[b u_{1} \mathrm{e}^{a\left(\tau_{2}-t_{2}\right)}+(\eta \lambda+\delta \epsilon)\left(u_{1}-\frac{\bar{u}}{2}\right)\right] \mathrm{e}^{\lambda \tau_{2}}, \\
\dot{u}^{*}\left(\tau_{2}-0\right)=-\frac{1}{\eta}\left[b u_{1} \mathrm{e}^{a\left(\tau_{2}-t_{2}\right)}+\delta \epsilon\left(u_{1}-\frac{\bar{u}}{2}\right)\right]<0 \Rightarrow b<0,
\end{array}\right.
$$

where $\dot{u}^{*}\left(\tau_{2}-0\right)$ denotes the left derivative of $\dot{u}^{*}(t)$ at $\tau_{2}$.

Finally by (29) and (31) one has

$$
\tau_{1}=t_{1} \quad \tau_{2}=t_{2} \Rightarrow D_{1}=\Delta_{1}(\lambda-a) / \Delta, \quad D_{2}=\Delta_{2}(\lambda+a) / \Delta,
$$

where

$$
\begin{aligned}
& \Delta=2 a \cdot \frac{\lambda^{2}(\delta \epsilon-a \eta)^{2} \mathrm{e}^{\lambda\left(t_{1}-t_{2}\right)}-(\lambda+a)^{2}(\delta \epsilon+\lambda \eta)^{2} \mathrm{e}^{\lambda\left(t_{2}-t_{1}\right)}}{\lambda(a+\lambda)(\delta \epsilon+\lambda \eta)}<0, \\
& \Delta_{1}=\left[2 a y_{1}+b \bar{u}+2 c\right] \mathrm{e}^{-\lambda t_{2}}-[(a+\lambda)(\delta \epsilon+\lambda \eta) \bar{u}] \mathrm{e}^{-\lambda t_{1}} / \lambda, \\
& \Delta_{2}=\left[2 a y_{1}+b \bar{u}+2 c\right] \mathrm{e}^{\lambda t_{2}}-\frac{\lambda b^{2} \bar{u}}{(a+\lambda)(\delta \epsilon+\lambda \eta)} \mathrm{e}^{\lambda t_{1}} .
\end{aligned}
$$

Remark that

$$
2 a y_{1}+b \bar{u}+2 c>0 \text {. }
$$


Indeed, if $b<0$ then (37) holds due to (11) and (9), if $b \geqslant 0$ then, using (9) one obtains $2 a y_{1}+b \bar{u}+2 c \geqslant$ $2 a\left(y_{1}+\eta \bar{u}\right)=2 a(\delta \bar{M}+\eta \bar{\alpha})>0$, i.e. (37) holds again.

To prove (28) we consider explicitly four cases:

(1) if $\tau_{1} \neq t_{1}$ and $\tau_{2} \neq t_{2}$ then $D_{1}$ and $D_{2}$ are negative due to (33) and (10); moreover also $b<0$ due to (35), therefore (28) holds;

(2) if $\tau_{1}=t_{1}$ and $\tau_{2} \neq t_{2}$ then due to (35) we have $b<0$ and, using the sign of $\dot{u}^{*}\left(\tau_{2}-0\right)$, also $D_{1}<0$; moreover since $b<0$ from (23) we obtain $C_{1}<0$. Using (29) and (37) and boundary condition $y\left(t_{1}\right)=y_{1}$ we have $C_{2}>0$, i.e. $D_{2}<0$ (see (23)); this way (28) holds;

(3) if $\tau_{1} \neq t_{1}$ and $\tau_{2}=t_{2}$ then using (10) from (34) we have that $D_{2}<0$, i.e. $C_{2}>0$ (see (23)); using (31) and the boundary condition $z\left(t_{2}\right)=0$ we get $C_{1}<0$ and also in this case the sign of $b$ is the same as the sign of $C_{2}$ since

$\operatorname{sign} b=\operatorname{sign}(a-\lambda)$;

(4) if $\tau_{1}=t_{1}$ and $\tau_{2}=t_{2}$ then we can use (36); by (37) and (38) we obtain again (28).

Proof of Proposition 3.1. Due to (5) we have that condition $\delta \leqslant \theta \eta /(\eta \gamma+\epsilon)$, the inefficient retailer, can be rewritten as $b \geqslant 0$. If $b \geqslant 0$ then $\tau_{2}$ must coincide with $t_{2}$ (see (35)). In this case, from Lemma B.2 we have

$$
u^{*}(t)= \begin{cases}u_{1}, & t \in\left[t_{1}, \tau_{1}\right), \\ D_{1} \mathrm{e}^{\lambda t}+D_{2} \mathrm{e}^{-\lambda t}+\bar{u} / 2, & t \in\left[\tau_{1}, t_{2}\right],\end{cases}
$$

where $D_{1} \geqslant 0$ and $D_{2}<0$ due to Lemma B.3; this means that $\alpha^{*}(t)$ satisfies (3). So function $u^{*}(t)$ is strictly increasing on $\left[\tau_{1}, t_{2}\right]$ and the same holds for $\alpha^{*}(t)$.

If instead $\delta>\theta \eta /(\eta \gamma+\epsilon)$, the efficient retailer case, we have $b<0$. In that case, by Lemma B.2,

$$
u^{*}(t)= \begin{cases}u_{1}, & t \in\left[t_{1}, \tau_{1}\right), \\ D_{1} \mathrm{e}^{\lambda t}+D_{2} \mathrm{e}^{-\lambda t}+\bar{u} / 2, & t \in\left[\tau_{1}, \tau_{2}\right], \\ u_{1}, & t \in\left(\tau_{2}, t_{2}\right],\end{cases}
$$

with $D_{1}<0$ and $D_{2}<0$ (see Lemma B.3); therefore (4) holds. Function $u^{*}(t)$ is strictly concave on $\left[\tau_{1}, \tau_{2}\right]$ and $\alpha^{*}(t)$ has the same property, therefore they are both quasi-concave on $\left[t_{1}, t_{2}\right]$.

Let us show now that if $b<0$ then $\alpha^{*}\left(\tau_{1}\right) \leqslant \alpha^{*}\left(\tau_{2}\right)$, i.e.

$$
u^{*}\left(\tau_{1}\right) \leqslant u^{*}\left(\tau_{2}\right) .
$$

Indeed, if $\tau_{1}>t_{1}$ then, of course, (41) holds, due to the structure of the optimal control $u^{*}(t)$ given by (40). When $\tau_{1}=t_{1}$ then, using (29), (31) we obtain, by straightforward calculations,

$$
u^{*}\left(\tau_{2}\right)-u^{*}\left(\tau_{1}\right)=u^{*}\left(\tau_{2}\right)-u^{*}\left(t_{1}\right)=\frac{(a+\lambda)\left[1+\mathrm{e}^{\lambda\left(t_{1}-\tau_{2}\right)}\right]\left[\mathrm{e}^{\lambda\left(\tau_{2}-t_{1}\right)}-1+\frac{2 \lambda}{a+\lambda}\right] A}{\eta\left[(a+\lambda)^{2} \mathrm{e}^{\lambda\left(\tau_{2}-t_{1}\right)}-(a-\lambda)^{2} \mathrm{e}^{\lambda\left(t_{1}-\tau_{2}\right)}\right]}
$$

where we have put $A=a y_{1}+c+b u_{1}\left(1-\mathrm{e}^{a\left(\tau_{2}-t_{2}\right)}\right)$. Due to (11) we have $A \geqslant 0$, and, $\operatorname{since} \operatorname{sign}\left(u^{*}\left(\tau_{2}\right)-u^{*}\left(\tau_{1}\right)\right)=\operatorname{sign} A$, (41) holds.

\section{References}

Basuroy, S., Mantrala, M., Walters, R.G., 2001. The impact of category management on retailer prices and performance: Theory and evidence. Journal of Marketing 65, 16-32.

Besanko, D., Dubé, J.-P., Gupta, S., 2005. Own-brand and cross-brand retail passthrough. Marketing Science 24, $123-137$.

Boulding, W., Staelin, R., 1993. A look on the cost side: Market share and the competitive environment. Marketing Science 12, $144-166$.

Buratto, A., Grosset, L., Viscolani, B., 2006. Advertising a new product in a segmented market. European Journal of Operational Research 175, 12621267.

Campbell, J., Pritchard, R., 1976. Motivation theory in industrial and organizational psychology. In: Dunnette, M. (Ed.), Handbook of Industrial and Organizational Psychology. Rand McNally, Chicago, pp. 62-130.

Churchill Jr., G.A., Ford, N.M., Hartley, S.W., Walker Jr., O.J., 1985. The determinants of salesperson performance: A meta-analysis. Journal of Marketing Research 22, 103-118.

Feichtinger, G., Hartl, R., Sethi, S., 1994. Dynamic optimal control models in advertising: Recent developments. Management Science 40, 195-226.

Gerstner, E., Hess, J.D., 1995. Pull promotions and channel coordination. Marketing Science 14, 43-60.

Grant, K., Cravens, D.W., Low, G.S., Moncrief, W.C., 2001. The role of satisfaction with territory design on the motivation, attitudes, and work outcomes of salespeople. Journal of the Academy of Marketing Science 29, 165-178.

Jørgensen, S., Zaccour, G., 2004. Differential Games in Marketing. Kluwer Academic Publishers, London. 
Miao, C.F., Evans, K.R., Zou, S., 2007. the role of salesperson motivation in sales control systems - Intrinsic and extrinsic motivation revisited. Journal of Business Research 60, 417-425.

Mitchell, T., 1982. Motivation: New directions for theory, research and practice. Academy of Management Review 7, 80-88.

Nerlove, M., Arrow, K., 1962. Optimal advertising policy under dynamic conditions. Economica 29, 129-142.

Osterloh, M., Frey, B., 2000. Motivation, knowledge transfer and organizational forms. Organization Science 11, 538-550.

Ouyang, Y., Daganzo, C., 2006. Characterization of the Bullwhip Effect in linear, time-invariant supply chains: Some formulae and tests. Management Science 52, 1544-1556.

Ray, S., Chen, H., Bergen, M., Levy, D., 2006. Asymmetric wholesale pricing: Theory and evidence. Marketing Science 25, $131-154$.

Rentz, J.O., Shepherd, C.D., Tashchian, A., Dabholkar, P.A., Ladd, R.T., 2002. A measure of selling skill: Scale development and validation. Journal of Personal Selling \& Sales Management 22, 13-21, Winter.

Seierstad, A., Sydsæter, K., 1987. Optimal Control Theory with Economic Applications. North-Holland, Amsterdam.

Sethi, S., Thompson, G., 2000. Optimal Control Theory: Applications to Management Science and Economics. Kluwer Academic Publishers, Boston.

Vidale, M.L., Wolfe, H.B., 1957. An operations research study for sales response to advertising. Operations Research 5, 370-381.

Vroom, V.H., 1964. Work and Motivation. Wiley, New York.

Walker Jr., O.J., Churchill Jr., G.A., Ford, N.M., 1977. Motivation and performance in industrial selling: Present knowledge and needed research. Journal of Marketing Research 14 (May), 156-168.

Walters, R.G., 1989. An empirical investigation into retailer response to manufacturer trade promotions. Journal of Retailing 65, $253-272$. 